\title{
REDUCTIVE ALGEBRAS CONTAINING A DIRECT SUM OF THE UNILATERAL SHIFT AND A CERTAIN OTHER OPERATOR ARE SELFADJOINT
}

\author{
MOHAMAD A. ANSARI ${ }^{1}$
}

\begin{abstract}
We give a partial solution of the reductive algebra problem to prove that: a reductive algebra containing the direct sum of a unilateral shift of finite multiplicity and a finite-dimensional completely nonunitary contraction is a von Neumann algebra.
\end{abstract}

1. Introduction. An algebra $\mathscr{U}$ of operators on a Hilbert space is reductive if it is weakly closed, contains the identity operator, and has the property that Lat $\mathscr{U}$ $=$ Lat $\mathscr{U}^{*}$. Von Neumann algebras are reductive; the reductive algebra problem (posed in [5]) is: Is every reductive algebra a von Neumann algebra? There are a number of partial solutions to this problem $[1,2,3,4,5,6$, Theorem 9.15 and 7].

By presenting a new technique in this paper, we generalize the result of [4] to prove that a reductive algebra containing the direct sum of a unilateral shift of finite multiplicity and a finite-dimensional completely nonunitary contraction is a von Neumann algebra. A consequence is a new proof of Burnside's Theorem. We conclude by presenting an open question which arises from our work.

2. Throughout we let $S \in \mathscr{L}(\mathscr{H})$ denote a unilateral shift of finite multiplicity, and $T \in \mathscr{L}(\mathscr{K})$ a finite-dimensional completely nonunitary contraction.

THEOREM. If $\mathscr{U}$ is a reductive algebra containing the operator $S \oplus T$ then $\mathscr{U}$ is a von Neumann algebra.

The proof requires the following known Lemma.

LEMMA [4]. If $\mathscr{U}$ is a reductive algebra, and if the span of the ranges of the finite rank operators in $\mathscr{U}$ is the entire space, then $\mathscr{U}$ is a von Neumann algebra.

Proof of The Theorem. We show that the hypothesis of the Theorem implies the hypothesis of the Lemma. To this end, let $P(\lambda)=\prod_{i=1}^{m}\left(\lambda-\lambda_{i}\right)$ be the characteristic polynomial of $T$. Since $T$ is a completely nonunitary contraction, it follows that

Received by the editors March 8, 1984.

1980 Mathematics Subject Classification. Primary 47C05, 47C15; Secondary 46C10.

${ }^{1}$ This research was supported in part by Grant No. MCS 82-01607 from the National Science Foundation. 
$\left|\lambda_{i}\right|<1$ for $i=1,2, \ldots, m$. Define the finite Blaschke product

$$
B(\lambda)=\prod_{i=1}^{m}\left(\lambda-\lambda_{i}\right)\left(1-\bar{\lambda}_{i} \lambda\right)^{-1} .
$$

It is easy to show that the operator $U=B(S)$ is unitarily equivalent to a unilateral shift of multiplicity $m n$ and

$$
B(S \oplus T)=B(S) \oplus B(T)=U \oplus 0 \in \mathscr{U} .
$$

Define the algebra

$$
\begin{aligned}
& \mathscr{A}=\{X \in \mathscr{L}(\mathscr{H}): \text { There are operators } Y \in \mathscr{L}(\mathscr{H}, \mathscr{K}) \\
& \text { and } \left.Z \in \mathscr{L}(\mathscr{K}) \text { such that }\left[\begin{array}{cc}
X & 0 \\
Y & Z
\end{array}\right] \in \mathscr{U}\right\} .
\end{aligned}
$$

We prove that $\mathscr{A}_{w}$, the closure of $\mathscr{A}$ in the weak operator topology of $\mathscr{L}(\mathscr{H})$, is a reductive algebra. To this end, let $X$ be an operator in $\mathscr{A}$, let $f$ be a nonzero vector in $\mathscr{H}$, and let $\varepsilon$ be a positive number. There exist operators $Y$ and $Z$ such that $\left[\begin{array}{ll}X & 0 \\ Y & Z\end{array}\right] \in \mathscr{U}$. From (1) we conclude that

$$
(U \oplus 0)\left[\begin{array}{ll}
X & 0 \\
Y & Z
\end{array}\right]=U X \oplus 0 \in \mathscr{U} .
$$

Since $\mathscr{U}$ is reductive, there exists an operator $A=\left(A_{i j}\right) \in \mathscr{U}$ such that

$$
\left\|\left(A-\left(X^{*} U^{*} \oplus 0\right)\right)(U f, 0)\right\|<\varepsilon ;
$$

hence

$$
\left\|\left(A_{11} U-X^{*}\right) f\right\|<\varepsilon .
$$

Since the operator $A_{11} U \in \mathscr{A}$, it is easy to conclude from (2) that Lat $\mathscr{A}=$ Lat $\mathscr{A}^{*}$. Therefore, $\mathscr{A}_{w}$ is a reductive algebra.

Now $\mathscr{A}_{w}$ is a reductive algebra containing $U$, a unilateral shift of finite multiplicity; thus $\mathscr{A}_{w}$ is a von Neumann algebra by [4, Theorem 1]. This implies that $U^{*} \in \mathscr{A}_{w}$; hence, there exists a net $\left\{X_{\alpha}\right\}_{\alpha} \subset \mathscr{A}$ such that

$$
U^{*}=\lim _{\alpha} X_{\alpha} \quad \text { (weak operator topology). }
$$

From the definition of $\mathscr{A}$, it follows that there are operators $Y_{\alpha}$ and $Z_{\alpha}$ such that

$$
\left[\begin{array}{cc}
X_{\alpha} & 0 \\
Y_{\alpha} & Z_{\alpha}
\end{array}\right] \in \mathscr{U} \quad \text { for every } \alpha .
$$

Thus we have

$$
(U \oplus 0)\left[\begin{array}{cc}
X_{\alpha} & 0 \\
Y_{\alpha} & Z_{\alpha}
\end{array}\right]=\left(U X_{\alpha} \oplus 0\right) \in \mathscr{U}
$$

for every $\alpha$. Now (3) and (4) together imply that

$$
U U^{*} \oplus 0=(1-p) \oplus 0 \in \mathscr{U} ;
$$

hence,

$$
F_{0}=P \oplus 1 \text { and } F_{k}=U^{k} P \oplus 0 \in \mathscr{U}, \quad \text { for } k \geqslant 1,
$$

where $P$ is the orthogonal projection of $\mathscr{H}$ onto ker $U^{*}$. It is easy to show that

$$
\bigvee_{k \geqslant 0} \operatorname{ran} F_{k}=\mathscr{H} \oplus \mathscr{K} \text {. }
$$


Since the operators $F_{k}, k \geqslant 0$, are of finite rank, it follows from (5) that the span of the ranges of all finite rank operators in $\mathscr{U}$ is the entire space. Therefore, the Lemma implies that $\mathscr{U}$ is a von Neumann algebra, which was to be shown

The following classical result is an easy consequence of the Theorem.

COROllary (BURNSIDE). If $\mathscr{K}$ is a finite-dimensional Hilbert space and $\mathscr{U}$ is a subalgebra of $\mathscr{L}(\mathscr{K})$ with no nontrivial subspace, then $\mathscr{U}=\mathscr{L}(\mathscr{K})$.

Proof. Since Lat $\mathscr{U}=\{\{0\}, \mathscr{K}\}$, it follows that $\mathscr{U}$ is a reductive algebra. Now let $S \in \mathscr{L}(\mathscr{H})$ be a unilateral shift of finite multiplicity, and define the reductive algebra $\mathscr{W}=\mathscr{L}(\mathscr{H}) \oplus \mathscr{U}$. Since $\mathscr{W}$ contains the operator $S \oplus 0, \mathscr{W}$ is a von Neumann algebra by the Theorem, which implies that $\mathscr{U}$ is a von Neumann algebra. It easily follows from this fact that $\mathscr{U}=\mathscr{U}^{\prime \prime}$, where $\mathscr{U}^{\prime \prime}$ is the double commutant of $\mathscr{U}$.

The fact that $\mathscr{U}$ has no nontrivial invariant subspace implies that $\mathscr{U}^{\prime}$, the commutant of $\mathscr{U}$, consists of the scalar operators. Therefore,

$$
\mathscr{U}=\mathscr{U}^{\prime \prime}=\left(\mathscr{U}^{\prime}\right)^{\prime}=\{\lambda 1: \lambda \in \mathbf{C}\}^{\prime}=\mathscr{L}(\mathscr{K}) .
$$

Thus $\mathscr{U}=\mathscr{L}(\mathscr{K})$, which was to be shown.

REMARK. If the Theorem remains true without the restriction that $\mathscr{K}$ be finite dimensional, then every reductive algebra is a von Neumann algebra. That is, the reductive algebra problem has a solution.

A completely nonunitary contraction $T \in \mathscr{L}(\mathscr{H})$ is a $C_{0}$-operator if there exists an inner function $f \in H^{\infty}(\mathrm{D})$ such that $f(T)=0 . T$ is an essentially unitary $C_{0}$-operator if the defect operators $\left(1-T^{*} T\right)_{\frac{1}{2}}$ and $\left(1-T T^{*}\right)^{\frac{1}{2}}$ are compact.

Question. If $S \in \mathscr{L}(\mathscr{H})$ is a unilateral shift of finite multiplicity, if $T \in \mathscr{L}(\mathscr{K})$ is an essentially unitary $C_{0}$-operator, and if $\mathscr{U}$ is a reductive algebra containing the operator $S \oplus T$, must $\mathscr{U}$ be a von Neumann algebra?

Acknowledgement. The author wishes to express his gratitude to Professor Allen Shields for his valuable guidance.

\section{REFERENCES}

1. E. Azoff, Compact operators in reductive algebras, Canad. J. Math. 27 (1975), 152-154.

2. K. J. Harrison, W. E. Longstaff and P. Rosenthal, Some tractable non-selfadjoint operator algebras, J. London Math. Soc. (2) 26 (1982), 325-330.

3. B. Moore, III and E. Nordgren, On transitive algebras containing $C_{0}$-operators, Indiana Univ. Math. J. 24 (1975), 777-789.

4. E. Nordgren and P. Rosenthal, Algebras containing unilateral shifts or finite rank operators, Duke Math J. 40 (1973), 419-424.

5. H. Radjavi and P. Rosenthal, A sufficient condition that an operator algebra be selfadjoint, Canad. J. Math. 23 (1971), 588-597.

6. _ Invariant subspaces, Springer-Verlag, 1972.

7. P. Rosenthal, On reductive algebras containing compact operators, Proc. Amer. Math. Soc. 47 (1975), $338-340$.

Department of Mathematics, Pennsylvania State University, Berks Campus, Reading, PennSYLVANIA 19608

Current address: 62 Medinah Drive, Reading, Pennsylvania 19607 